International Journal of Electrical Engineering and Technology (IJEET)

Volume 12, Issue 5, May 2021, pp. 96-108, Article ID: IJEET_12_05_009

Available online at https://iaeme.com/Home/issue/IJEET?Volume $=12 \&$ Issue $=5$

ISSN Print: 0976-6545 and ISSN Online: 0976-6553

DOI: 10.34218/IJEET.12.5.2021.009

(C) IAEME Publication

Scopus Indexed

\title{
SPECTRAL AND PERFORMANCE MEASURES ANALYSIS OF ECG SIGNAL USING VARIOUS TRANSFORMS AND DIFFERENT TYPES OF IIR AND FIR FILTERS WITH DIFFERENT ORDERS
}

\section{Dr. P. Kannan}

Associate Professor and Head, Department of Electronics and Communication Engineering, Amrita College of Engineering and Technology, Nagercoil, Tamilnadu, India

\section{S. Maheswari}

Assistant Professor, Department of Electronics and Communication Engineering, Amrita College of Engineering and Technology, Nagercoil, Tamilnadu, India

\section{A. Pon Bharathi}

Assistant Professor, Department of Electronics and Communication Engineering, Amrita College of Engineering and Technology, Nagercoil, Tamilnadu, India

Allan J Wilson

Assistant Professor, Department of Electronics and Communication Engineering, Amrita College of Engineering and Technology, Nagercoil, Tamilnadu, India

\begin{abstract}
The electrical exercises of the heart are addressed as waves, which demonstrate the pinnacles and spikes of ECG signal. The input ECG signal is mixed with AWGN commotion. The target of the proposed framework is to evaluate the input ECG signal for different transforms like DCT, DWT, FWHT and FFT and afterward the extricated signal are filtered utilizing two significant filtering procedures like IIR and FIR. The filtering methods are applied under various classes for each channel to be specific IIR and FIR. In which IIR incorporates the Butterworth, Chebyshev type I and II and Elliptic channel. The FIR is typically performed utilizing windowing methods like Kaiser, Chebyshev and hamming windows. The examination is made for the FIR and IIR techniques utilizing the presentation measurements for example, time utilization, order and signal to noise ratio.
\end{abstract}

Key words: Windowing techniques, Transforms, ECG signal, IIR Filter, FIR Filter, and AWGN Noise 
Cite this Article: P. Kannan, S. Maheswari, A. Pon Bharathi and Allan J Wilson, Spectral and Performance Measures Analysis of ECG Signal Using Various Transforms and Different Types of IIR and FIR Filters with Different Orders, International Journal of Electrical Engineering and Technology (IJEET), 12(5), 2021, pp. 96-108. https://iaeme.com/Home/issue/IJEET?Volume=12\&Issue $=5$

\section{INTRODUCTION}

The fundamental job of ECG is to break down the working of the heart by means of the P, Q, $\mathrm{R}, \mathrm{S}, \mathrm{T}$ waves that were estimated with the electrodes set at various parts of the body. A typical ECG has 10 electrodes and 12 leads which are put on the skin. The nature and deviations from the standard healthy wave driving in separation of different dis-functionality present in the heart. The different IIR filtering procedures incorporates Butterworth filter, Chebyshev type I filter, Chebyshev type II filter and Elliptic filter. The FIR filtering methods incorporates windowing strategies. The different windowing methods are Hamming window, Hamming window, Kaiser Window, Chebyshev window, Rectangular window, Blackman window, etc.

\section{PROPOSED SYSTEM}

The separated signals from the transforms are to be filtered for which different filtering methods such as IIR and FIR are utilized. These filters can isolate the noise from the information ECG signal which can be prepared for the few clinical examination. The eventual outcomes acquired from the filtering strategies are contrasted and each other dependent on execution measurements, for example, time utilization, order of the filter and signal to noise ratio.

\subsection{Transforms}

The transforms are utilized to deteriorate a signal and extraction of those signals can improve its presentation measurements. The various kinds of transforms utilized here are talked about beneath.

\subsubsection{DCT (Discrete Cosine Transform)}

A Discrete Cosine Transform (DCT) express a movement of limitedly various data focuses as far as an entirety of cosine limits fluctuate at various frequencies.

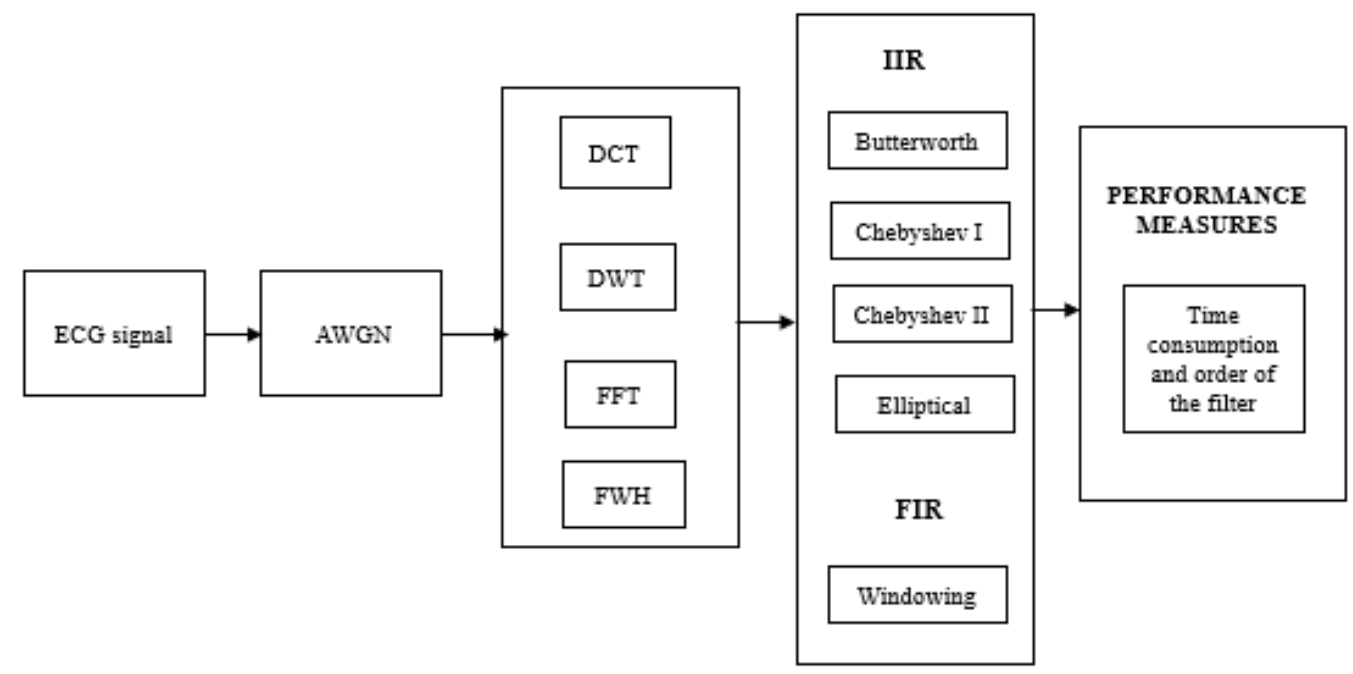

Figure.1 Process of proposed system using IIR and FIR filters 
Discrete Cosine Transform of $\mathrm{N}$ sample is characterized as

$$
w(k)=\frac{1}{\sqrt{N}}
$$

\subsubsection{FFT (Fast Fourier Transform)}

The FFT is progressed algorithm of Fourier transform which is mostly utilized in deciding the discrete form of the fourier transform and reverse function is likewise conceivable. FFT algorithms principally rely upon the factorization of $\mathrm{N}$.

The coefficient of sequence of $\mathrm{N}$ for $\mathrm{u}^{\text {th }}$ FFT is given as

$$
X_{k}=\sum_{n=0}^{N-1} x_{n} e^{-i 2 \pi n k / N} \quad ; k=0,1, \ldots . N-1
$$

\subsubsection{Wavelet Transform}

The wavelet transforms are utilized to breakdown the single wavelet into sub wavelet from which data can be extracted. For discrete examining, a calculation named Discrete Wavelet Transform is utilized.

\subsubsection{Fast Walsh Hadamard Transform (FWHT)}

The significant trait of Walsh function is deciding the quantity of zero intersection for each unit time interval. Every single Walsh function has sequence esteem. The utilizations of Walsh function are spectrum analysis, filtering, speech and medical signal processing, multiplexing and coding in communications.

The length $\mathrm{N}$ of a Walsh transform for a signal $\mathbf{X}[\mathbf{k}]$ is

$$
\mathrm{X}[\mathrm{k}]=\sum_{\mathrm{m}=0}^{\mathrm{N}-1} \mathrm{x}[\mathrm{m}] \prod_{\mathrm{i}=0}^{\mathrm{n}-1}(-1)^{\left(\mathrm{k}_{\mathrm{i}}+\mathrm{k}_{\mathrm{i}+1}\right) \mathrm{m}_{\mathrm{n}-1-\mathrm{i}}}
$$

\subsubsection{Additive White Gaussian Noise (AWGN)}

AWGN is a fundamental commotion which is utilized to imitate normally

\subsection{Filters}

The undesirable or noise segments are eliminated from the signal utilizing various kinds of filters. The filters are ordered dependent on the linearity, causality, time variations IIR, FIR and simple or digital signal.

\subsubsection{IIR Filters}

IIR filters possess an infinite impulse response for digital filters. The various types of IIR filters are Butterworth filter, Chebyshev type I, II filters, Elliptic filter.

\subsubsection{Butterworth filter}

The Butterworth filter is another sort of signal processing filter proposed to have level repeat response in the pass band ie, flat frequency reaction. It is in like manner implied as a maximum level extent channel.

The frequency response of Butterworth filter given by,

$$
H(j \omega)=\frac{1}{\sqrt{1+\left(\frac{\omega}{\omega_{\mathrm{c}}}\right)^{2 \mathrm{n}}}}
$$




\subsubsection{Chebyshev Type I Filter}

The magnitude response of the Chebyshev low pass filter is discussed below

$$
|\mathbf{H}(\mathrm{j} \Omega)|=\frac{A}{\left[1+\varepsilon^{2} \mathrm{C}_{\mathrm{N}}^{2}\left(\frac{\Omega}{\Omega_{\mathrm{c}}}\right)\right]^{0.5}}
$$

\subsubsection{Chebyshev Type II Filter}

The magnitude response of the filter is provided below and the Chebyshev type II is also called is inverse Chebyshev filter.

$$
|H(j \Omega)|=\frac{\varepsilon C_{N}\left(\frac{\Omega_{2}}{\Omega}\right)}{\left[1+\varepsilon^{2} C_{N}^{2}\left(\frac{\Omega_{2}}{\Omega}\right)\right]^{0.5}}
$$

\subsubsection{Elliptic Filter}

An elliptic filter is a signal processing filter with leveled swell (equiripple) conduct in both the passband and the stopband. The measure of swell in each band is independently amendable. The gain of a low pass elliptic filter as an element of rakish recurrence $\omega$ is given by

$$
G_{n}(\omega)=\frac{1}{\sqrt{1+\epsilon^{2} R_{n}^{2}\left(\xi, \frac{\omega}{\omega_{0}}\right)}}
$$

\subsubsection{FIR Filters}

The different windowing procedures that goes under FIR filters are Kaiser window, Hamming window, Hamming window, Rectangular window, Chebyshev window, Blackman window.

\subsubsection{Kaiser window}

The windowing is the significant technique for signal processing. It is represented as

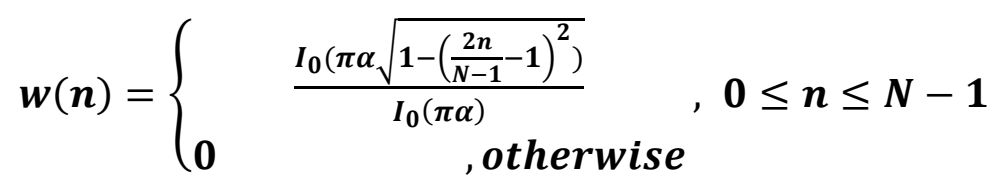

\subsubsection{Hamming window}

The hamming window is given as

$$
\mathbf{w}(\mathbf{n})=.54-.46 \cos (2 \pi n \mid \mathbf{N}-1)
$$

The Hamming window is represented mathematically as

$$
\mathbf{w}(\mathbf{n})=.5-.5 \cos (2 \pi n \mid N-1)
$$

\section{RESULTS AND DISCUSSION}

The accompanying are the results that are obtained by using MATLAB programming. ECG signal is taken as information and after that it is mixed with AWGN commotion. The info ECG signal is applied for various transforms, for example, DCT, DWT, FFT, FWHT and afterward filtered utilizing IIR and FIR. By then assessments are put to the side as time taken, SNR and cross correlation. 


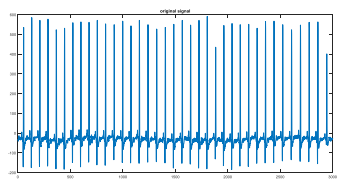

Figure.2 ECG signal

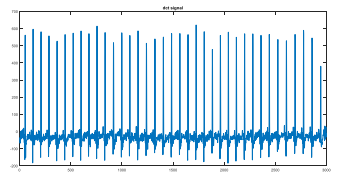

Figure.4 DCTblended signal

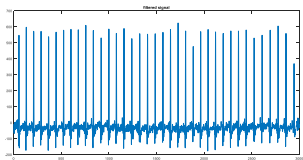

Figure.6 Filtered using Butterworth filter (low pass) of order 3

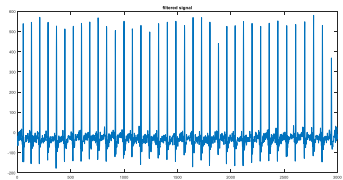

Figure.8 Filtered using Chebyshev Type I filter (low pass) of order 2

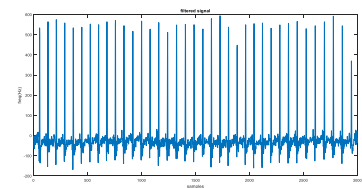

Figure.10 Filtered using Chebyshev Type I filter (low pass) of order 5

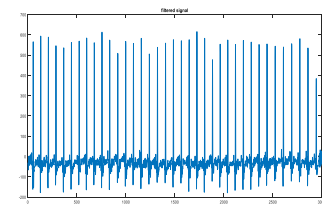

Figure.12 Filtered using Chebyshev Type II filter (low pass) of order 3

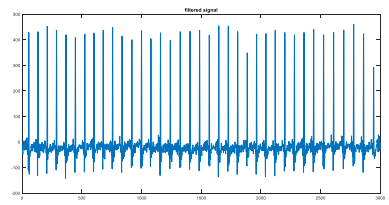

Figure.14 Filtered using Elliptic filter (low pass) of order 2

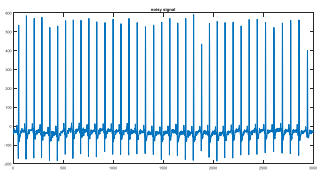

Figure.3 Input ECG signal blended with AWGN noise

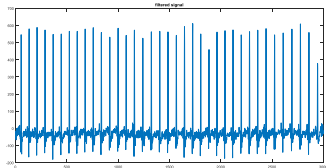

Figure.5 Filtered using Butterworth filter (low pass) of order 2

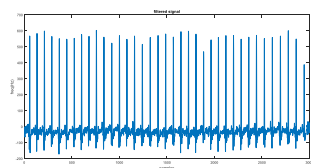

Figure.7 Filtered using Butterworth filter (low pass) of order 5

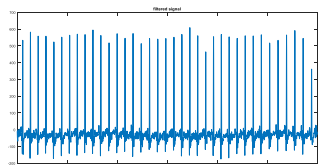

Figure.9 Filtered using Chebyshev Type I filter (low pass) of order 3

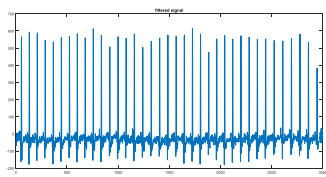

Figure.11 Filtered using Chebyshev Type II filter (low pass) of order 2

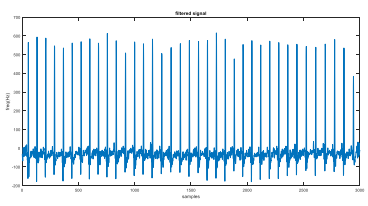

Figure.13 Filtered using Chebyshev Type II filter (low pass) of order 5

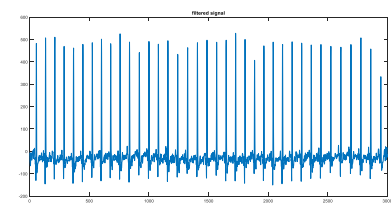

Figure.15 Filtered using Elliptic filter (low pass) of order 3 
Spectral and Performance Measures Analysis of ECG Signal Using Various Transforms and Different Types of IIR and FIR Filters with Different Orders

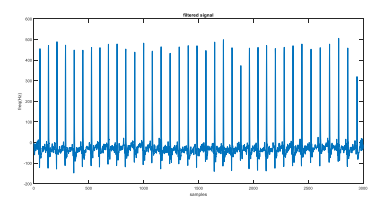

Figure.16 filtered using Elliptic filter (low pass) of order 5

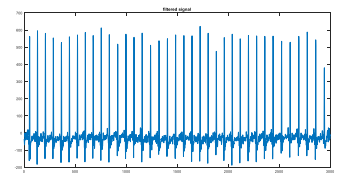

Figure.18 Filtered using Hamming window of order 10

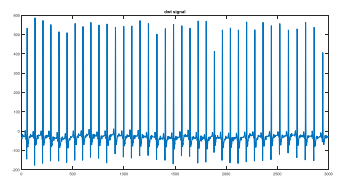

Figure.20 Input ECG signal blended with noise for DWT

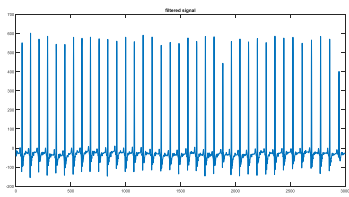

Figure.22 Filtered using Butterworth filter (low pass) of order 3

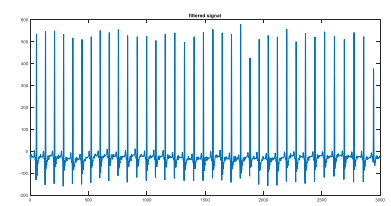

Figure.24 Filtered using Chebyshev Type I filter (low pass) of order 2

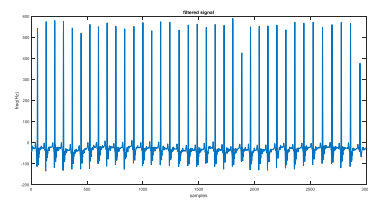

Figure.26 Filtered using Chebyshev Type I filter (low pass) of order 5

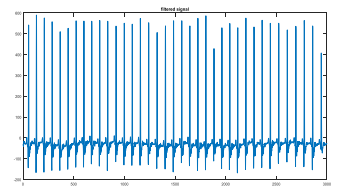

Figure.28 Filtered using Chebyshev Type II filter (low pass) of order 3

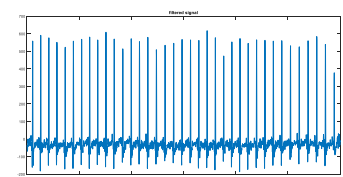

Figure.17 Filtered using Kaiser window of order 10

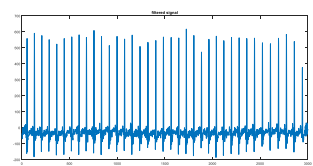

Figure.19 Filtered using Chebyshev window of order 34

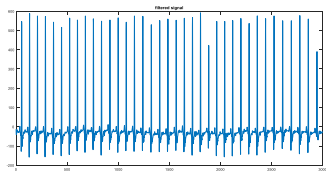

Figure.21 Filtered using Butterworth filter (low pass) of order 2

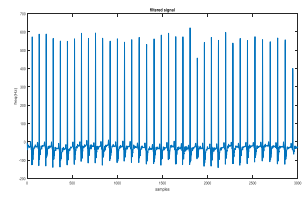

Figure.23 Filtered using Butterworth filter (low pass) of order 5

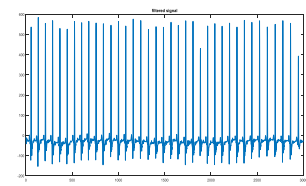

Figure.25 Filtered using Chebyshev Type I filter (low pass) of order 3

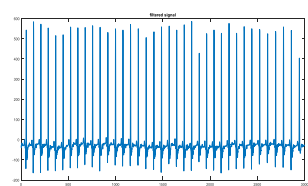

Figure.27 Filtered using Chebyshev Type II filter (low pass) of order 2

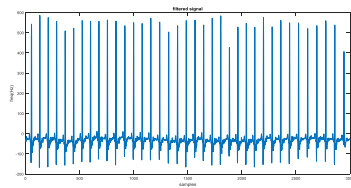

Figure.29 Filtered using Chebyshev Type II filter (low pass) of order 5 


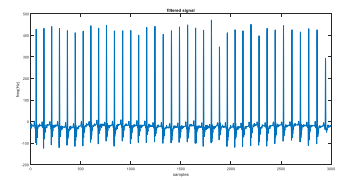

Figure.30 Filtered using Elliptic filter (low pass) of order 2

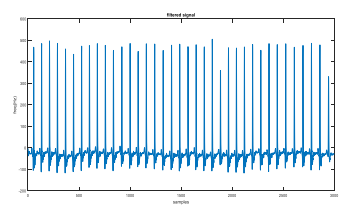

Figure.32 Filtered using Elliptic filter (low pass) of order 5

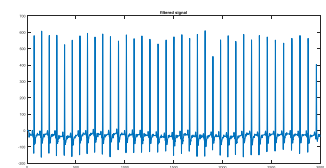

Figure.34 Filtered using Hamming window of order 10

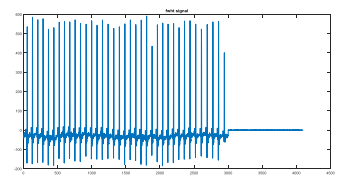

Figure.36 ECG signal blended noise for FWHT

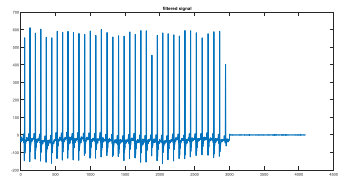

Figure.38 Filtered using Butterworth filter (low pass) of order 3

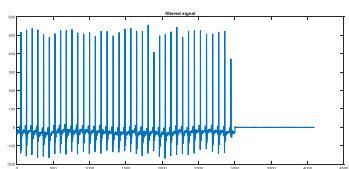

Figure.40 Filtered using Chebyshev Type I filter (low pass) of order 2

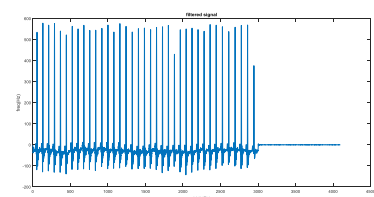

Figure.42 Filtered using Chebyshev Type I filter (low pass) of order 5

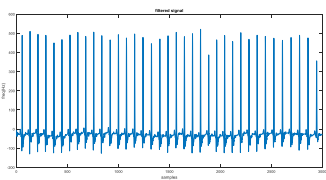

Figure.31 Filtered using Elliptic filter (low pass) of order 3

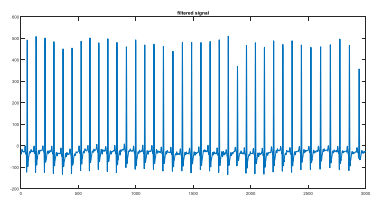

Figure.33 Filtered using Kaiser window of order 10

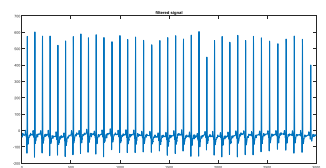

Figure.35 Filtered using Chebyshev window of order 34

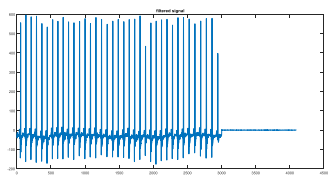

Figure.37 Filtered using Butterworth filter (low pass) of order 2

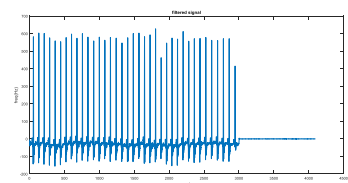

Figure.39 Filtered using Butterworth filter (low pass) of order 5

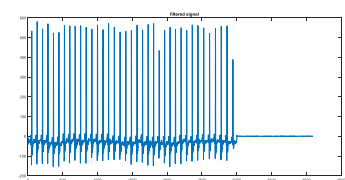

Figure.41 Filtered using Chebyshev Type I filter (low pass) of order 3

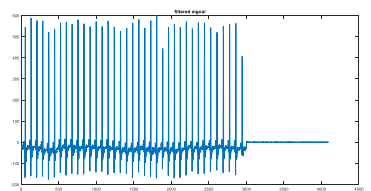

Figure.43 Filtered using Chebyshev Type II filter (low pass) of order 2 
Spectral and Performance Measures Analysis of ECG Signal Using Various Transforms and Different Types of IIR and FIR Filters with Different Orders

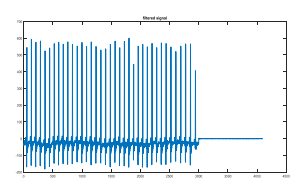

Figure.44 Filtered using Chebyshev Type II filter (low pass) of order 3

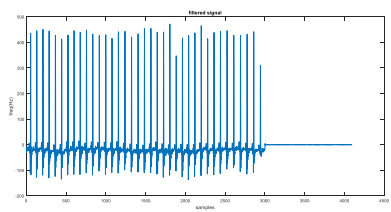

Figure.46 Filtered using Elliptic filter (low pass) of order 2

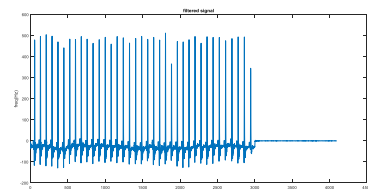

Figure.48 Filtered using Elliptic filter (low pass) of order 5

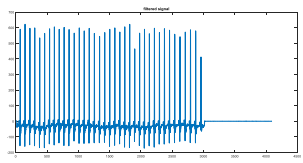

Figure.50 Filtered using Hamming window of order 10

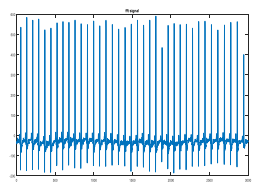

Figure.52 Input ECG signal blended noise for FFT

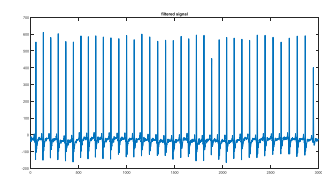

Figure.54 Filtered using Butterworth filter (low pass) of order 3

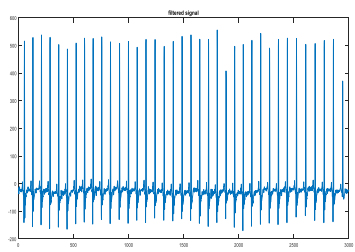

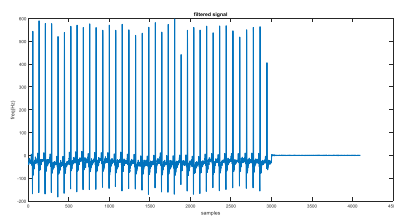

Figure.45 Filtered using Chebyshev Type II filter (low pass) of order 5

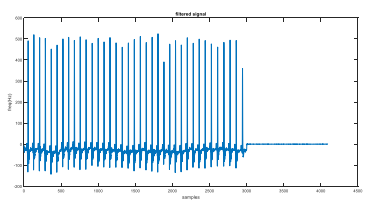

Figure.47 Filtered using Elliptic filter (low pass) of order 3

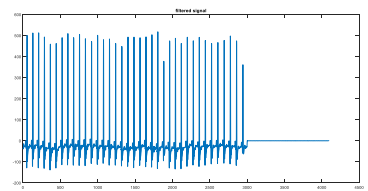

Figure.49 Filtered using Kaiser window of order 10

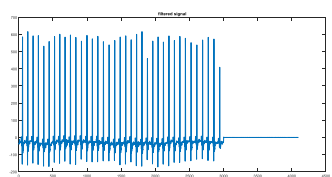

Figure.51 Filtered using Chebyshev window of order 34

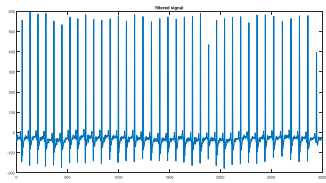

Figure.53 Filtered using Butterworth filter (low pass) of order 2

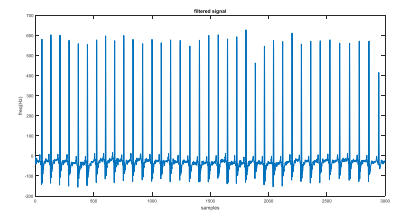

Figure.55. Filtered using Butterworth filter (low pass) of order 5

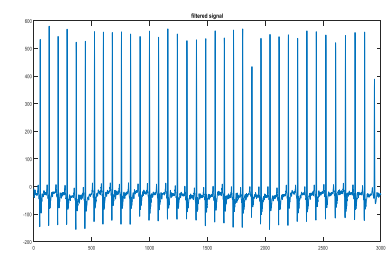


Figure.56 Filtered using Chebyshev filter Type I (low pass) of order 2

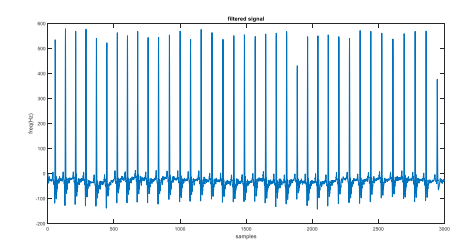

Figure.58 Filtered using Chebyshev filter Type I (low pass) of order 5

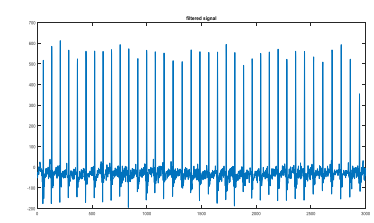

Figure.60 Filtered using Chebyshev filter Type II (low pass) of order 3

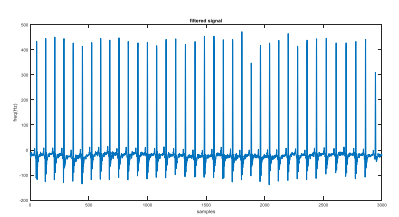

Figure.62 Filtered using Elliptic filter (low pass) of order 2

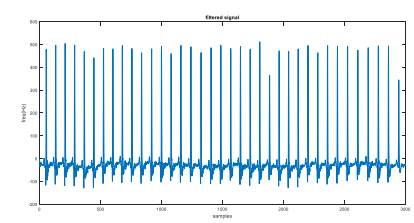

Figure.64 Filtered using Elliptic filter (low pass) of order 5

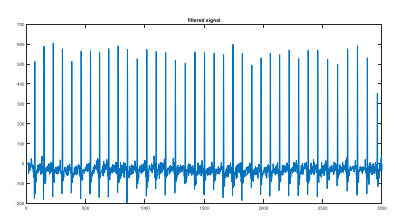

Figure.66 Filtered using Hamming window of order 10
Figure.57 Filtered using Chebyshev filter Type I (low pass) of order 3

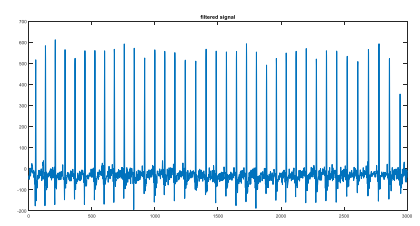

Figure.59 Filtered using Chebyshev filter Type II (low pass) of order 2

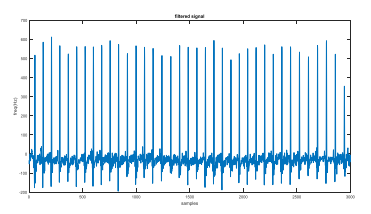

Figure.61 Filtered using Chebyshev filter Type II (low pass) of order 5

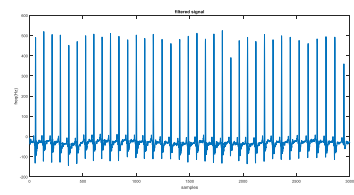

Figure.63 Filtered using Elliptic filter (low pass) of order 3

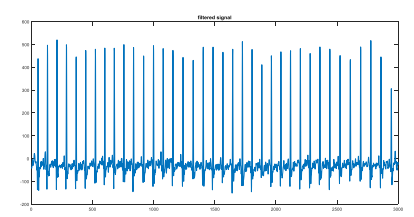

Figure.65 Filtered using Kaiser window of order 10

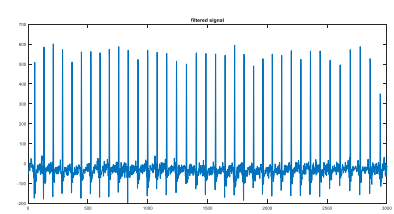

Figure.67Filtered using Chebyshev window of order 34 
Spectral and Performance Measures Analysis of ECG Signal Using Various Transforms and Different Types of IIR and FIR Filters with Different Orders

Table 1 Comparison of different transforms filtered by IIR filtering techniques of order 2

\begin{tabular}{|c|c|c|c|c|c|}
\hline Transform & Parameters & $\begin{array}{c}\text { Butterworth } \\
\text { filter(Low) }\end{array}$ & $\begin{array}{l}\text { Chebyshev } \\
\text { Type I(Low) }\end{array}$ & $\begin{array}{c}\text { Chebyshev } \\
\text { Type II(Low) }\end{array}$ & Elliptic Filter \\
\hline \multirow[t]{5}{*}{ DCT } & Time taken & 0.5084 & 0.5145 & 0.5848 & 0.5528 \\
\hline & PSD after filtering & 4.2133 & 3.7780 & 4.2145 & 2.1700 \\
\hline & $\begin{array}{l}\text { PSD before } \\
\text { filtering }\end{array}$ & 4.2096 & 4.2095 & 4.2095 & 4.2096 \\
\hline & SNR & 0.2313 & 0.6101 & 0.0980 & 2.6535 \\
\hline & Cross correlation & $3.6018 \mathrm{e}+07$ & $3.4302 \mathrm{e}+07$ & $3.5607 \mathrm{e}+07$ & $1.4704 \mathrm{e}+07$ \\
\hline \multirow[t]{5}{*}{ DWT } & Time taken & 3.0427 & 0.6913 & 0.0104 & 1.9836 \\
\hline & PSD after filtering & 4.3073 & 3.7729 & 4.2087 & 2.1671 \\
\hline & $\begin{array}{l}\text { PSD before } \\
\text { filtering }\end{array}$ & 4.2095 & 4.2096 & 4.2094 & 4.2096 \\
\hline & SNR & 0.0018 & 0.6254 & 0.1658 & 2.7085 \\
\hline & Cross correlation & $3.50 \mathrm{e}+07$ & $3.26 \mathrm{e}+07$ & $3.601 \mathrm{e}+07$ & $1.9455 \mathrm{e}+14$ \\
\hline \multirow[t]{5}{*}{ FFT } & Time taken & 0.5132 & 0.5169 & 0.4720 & 0.8472 \\
\hline & PSD after filtering & 4.2079 & 3.807 & 4.1158 & 2.1673 \\
\hline & $\begin{array}{l}\text { PSD before } \\
\text { filtering }\end{array}$ & 4.2096 & 4.2095 & 4.2095 & 4.2095 \\
\hline & SNR & 0.1561 & 0.9120 & 0.0142 & 2.5404 \\
\hline & Cross correlation & $3.60 \mathrm{e}+07$ & $3.00 \mathrm{e}+07$ & $3.621 \mathrm{e}+07$ & $2.0223 \mathrm{e}+07$ \\
\hline \multirow[t]{5}{*}{ FWHT } & Time taken & 3.6701 & 0.6238 & 0.6934 & 0.8393 \\
\hline & PSD after filtering & 3.0823 & 2.2461 & 3.0828 & 1.5875 \\
\hline & $\begin{array}{l}\text { PSD before } \\
\text { filtering }\end{array}$ & 4.2096 & 4.2094 & 4.2095 & 4.2096 \\
\hline & SNR & 0.5462 & 0.9661 & 0.1005 & 2.5405 \\
\hline & Cross correlation & $5.69 e+07$ & $2.10 \mathrm{e}+07$ & $3.75 \mathrm{e}+07$ & $2.0223 e+07$ \\
\hline
\end{tabular}

Table 2 Comparison of different transforms filtered by IIR filtering techniques of order 3

\begin{tabular}{|c|c|c|c|c|c|}
\hline Transform & Parameters & $\begin{array}{l}\text { Butterworth } \\
\text { filter(Low) }\end{array}$ & $\begin{array}{l}\text { Chebyshev } \\
\text { Type I(Low) }\end{array}$ & $\begin{array}{c}\text { Chebyshev } \\
\text { Type II(Low) }\end{array}$ & Elliptic Filter \\
\hline \multirow[t]{5}{*}{ DCT } & Time taken & 0.7454 & 0.8445 & 0.6264 & 0.5639 \\
\hline & PSD after filtering & 4.2137 & 4.1536 & 4.2144 & 3.7935 \\
\hline & $\begin{array}{l}\text { PSD before } \\
\text { filtering }\end{array}$ & 4.2094 & 4.2096 & 4.2094 & 4.2096 \\
\hline & SNR & 0.1111 & 0.3445 & 0.0072 & 1.1258 \\
\hline & Cross correlation & $3.5381 \mathrm{e}+07$ & $3.4076 e+07$ & $3.5414 \mathrm{e}+07$ & $2.8010 \mathrm{e}+07$ \\
\hline \multirow[t]{5}{*}{ DWT } & Time taken & 0.0768 & 0.8756 & 0.6103 & 0.8741 \\
\hline & PSD after filtering & 4.2076 & 4.1476 & 4.2081 & 3.7877 \\
\hline & $\begin{array}{l}\text { PSD before } \\
\text { filtering }\end{array}$ & 4.2095 & 4.2096 & 4.2096 & 4.2096 \\
\hline & SNR & 0.1633 & 0.3167 & 3.1490 & 1.1501 \\
\hline & Cross correlation & $3.49 \mathrm{e}+07$ & $3.37 e+07$ & $3.5 \mathrm{e}+07$ & $2.7853 e+07$ \\
\hline \multirow[t]{5}{*}{ FFT } & Time taken & 0.6221 & 0.4116 & 0.5114 & 0.8542 \\
\hline & PSD after filtering & 4.2080 & 4.0825 & 0.1158 & 3.7881 \\
\hline & $\begin{array}{l}\text { PSD before } \\
\text { filtering }\end{array}$ & 4.2094 & 4.2095 & 4.2095 & 4.2095 \\
\hline & SNR & 0.2448 & 0.7572 & 0.2504 & 1.0468 \\
\hline & Cross correlation & $4.231 \mathrm{e}+07$ & $3.34 \mathrm{e}+07$ & $6.23 e+07$ & $2.8524 \mathrm{e}+07$ \\
\hline \multirow[t]{5}{*}{ FWHT } & Time taken & 4.2303 & 0.8679 & 0.6859 & 0.9299 \\
\hline & PSD after filtering & 3.0828 & 2.9909 & 3.0828 & 2.7757 \\
\hline & $\begin{array}{l}\text { PSD before } \\
\text { filtering }\end{array}$ & 4.2095 & 4.2094 & 4.2095 & 4.2095 \\
\hline & SNR & 0.1250 & 0.2573 & 0.1175 & 1.0469 \\
\hline & Cross correlation & $3.781 \mathrm{e}+07$ & $3.45 e+07$ & $3.56 e+07$ & $2.8523 \mathrm{e}+07$ \\
\hline
\end{tabular}


Table 3 Comparison of different transforms filtered by IIR filtering techniques of order 5

\begin{tabular}{|c|c|c|c|c|c|}
\hline Transform & Parameters & $\begin{array}{l}\text { Butterworth } \\
\text { filter(Low) }\end{array}$ & $\begin{array}{c}\text { Chebyshev } \\
\text { Type I(Low) }\end{array}$ & $\begin{array}{l}\text { Chebyshev } \\
\text { Type II(Low) }\end{array}$ & Elliptic Filter \\
\hline \multirow[t]{5}{*}{ DCT } & Time taken & 0.7120 & 0.8043 & 0.9776 & 0.6586 \\
\hline & PSD after filtering & 4.2133 & 4.0615 & 4.2145 & 3.4935 \\
\hline & $\begin{array}{l}\text { PSD before } \\
\text { filtering }\end{array}$ & 4.2096 & 4.2096 & 4.2094 & 4.2096 \\
\hline & SNR & 0.1073 & 0.3730 & 0.1071 & 1.5218 \\
\hline & Cross correlation & $3.5413 \mathrm{e}+07$ & $3.3311 \mathrm{e}+07$ & $3.414 \mathrm{e}+07$ & $2.5569 \mathrm{e}+07$ \\
\hline \multirow[t]{5}{*}{ DWT } & Time taken & 0.7124 & 0.9040 & 0.9262 & 1.1587 \\
\hline & PSD after filtering & 4.2061 & 4.0540 & 4.2089 & 3.4875 \\
\hline & $\begin{array}{l}\text { PSD before } \\
\text { filtering }\end{array}$ & 4.2094 & 4.2094 & 4.2095 & 4.2094 \\
\hline & SNR & 0.1598 & 0.4179 & 0.1480 & 1.5400 \\
\hline & Cross correlation & $3.4988 \mathrm{e}+07$ & $3.2969 \mathrm{e}+07$ & $3.5082 \mathrm{e}+07$ & $2.5462 \mathrm{e}+07$ \\
\hline \multirow[t]{5}{*}{ FFT } & Time taken & 0.7772 & 0.8272 & 0.6289 & 0.9384 \\
\hline & PSD after filtering & 4.2068 & 3.8969 & 4.1159 & 3.4881 \\
\hline & $\begin{array}{l}\text { PSD before } \\
\text { filtering }\end{array}$ & 4.2096 & 4.2094 & 4.2095 & 4.2096 \\
\hline & SNR & 0.0206 & 0.5485 & 0.1404 & 1.4343 \\
\hline & Cross correlation & $3.6127 e+07$ & $3.1992 \mathrm{e}+07$ & $3.5144 e+07$ & $2.6089 \mathrm{e}+07$ \\
\hline \multirow[t]{5}{*}{ FWHT } & Time taken & 0.8435 & 0.8150 & 0.7849 & 0.8974 \\
\hline & PSD after filtering & 3.0828 & 2.8566 & 3.0828 & 2.5567 \\
\hline & $\begin{array}{l}\text { PSD before } \\
\text { filtering }\end{array}$ & 4.3096 & 4.2094 & 4.3095 & 4.2096 \\
\hline & SNR & 0.0207 & 0.5485 & 0.0075 & 1.4343 \\
\hline & Cross correlation & $3.6126 e+06$ & $3.1992 \mathrm{e}+07$ & $0.6236 e+07$ & $2.6089 e+07$ \\
\hline
\end{tabular}

Table 4 Comparison of different transforms filtered by FIR filtering techniques

\begin{tabular}{|c|c|c|c|c|}
\hline Transform & Parameters & Kaiser Window & Hamming Window & $\begin{array}{c}\text { Chebyshev } \\
\text { Window }\end{array}$ \\
\hline \multirow{5}{*}{ DCT } & Time taken & 0.3423 & 0.2357 & 0.2433 \\
\hline & PSD after filtering & 4.294 & 4.1381 & 4.1962 \\
\hline & PSD before filtering & 4.2096 & 4.3096 & 4.3096 \\
\hline & SNR & 0.8594 & 0.6423 & 0.1639 \\
\hline & Cross correlation & $2.89 \mathrm{e}+07$ & $3.08 \mathrm{e}+07$ & $3.4954 \mathrm{e}+72$ \\
\hline \multirow[t]{5}{*}{ DWT } & Time taken & 0.5168 & 1.0961 & 0.5616 \\
\hline & PSD after filtering & 4.2765 & 4.1476 & 4.1908 \\
\hline & PSD before filtering & 4.2096 & 4.3095 & 4.3095 \\
\hline & SNR & 0.8718 & 0.4490 & 0.2272 \\
\hline & Cross correlation & $2.67 \mathrm{e}+07$ & $2.2733 \mathrm{e}+07$ & $2.44 \mathrm{e}+45$ \\
\hline \multirow[t]{5}{*}{ FFT } & Time taken & 0.1756 & 0.1572 & 0.187 \\
\hline & PSD after filtering & 4.1921 & 4.0574 & 4.098 \\
\hline & PSD before filtering & 4.2096 & 4.2094 & 4.2095 \\
\hline & SNR & 0.8809 & 0.4245 & 0.0195 \\
\hline & Cross correlation & $2.69 \mathrm{e}+45$ & $2.2918 \mathrm{e}+12$ & $2.47 \mathrm{e}+10$ \\
\hline \multirow[t]{5}{*}{ FWHT } & Time taken & 0.3399 & 0.3902 & 0.2262 \\
\hline & PSD after filtering & 3.1408 & 3.0545 & 3.0697 \\
\hline & PSD before filtering & 4.2095 & 4.2096 & 4.2095 \\
\hline & SNR & 0.9045 & 0.3357 & 0.1029 \\
\hline & Cross correlation & $3.26 \mathrm{e}+07$ & $3.3599 \mathrm{e}+12$ & $3.45 \mathrm{e}+45$ \\
\hline
\end{tabular}

\section{CONCLUSIONS}

Discrete Cosine Transform and Discrete Wavelet Transform are applied to this noisy info ECG signal respectively. At that point the noise from the ECG signal is taken out by utilizing IIR and FIR filtering methods. In this paper, AWGN is added with this info ECG signal. Then different 
Spectral and Performance Measures Analysis of ECG Signal Using Various Transforms and Different Types of IIR and FIR Filters with Different Orders

transforms like Fast Fourier Transform, Fast Walsh Hadamard Transform, Discrete Cosine Transform incorporates Butterworth channel, Chebyshev Type 1filter, Chebyshev Type 2 channel, Elliptic filter of orders 2, 3, 5. FIR filtering strategies incorporates windowing techniques. After the commotion from the ECG signal is sifted by utilizing IIR and FIR filtering procedures the parameters like time utilization SNR, PSD before and after filtering, cross correlation are determined for various orders. From the boundaries it is tracked down that in case of IIR filtering techniques, DWT with Chebyshev type 1 filter of order 2 eliminates the noise better when contrasted and other transforms. Since it has the cross relationship esteem as 3.18e+07 and SNR esteem as 0.5654. The time taken by this transform is 0.6913. In case of FIR filtering techniques, DWT with Kaiser Window of request 10 eliminates the noise better in light of the fact that it has the cross correlation estimation of $2.97 \mathrm{e}+07$, SNR estimation of 0.8718 and time taken is 0.4169 . In future, IIR filtering techniques of higher orders and FIR filtering techniques with different windows like Hanning window, Blackman window, Rectangular window can be utilized for the expulsion of undesirable noise from the ECG signal.

\section{REFERENCES}

[1] Ankit Gupta,(2015),"ECG noise reduction by different filters - A Comparative Analysis", International Journal of Research in Computer and Communication Technology, Vol 4, Issue 7, ISSN(online) 2278-5841, ISSN(print) 2320-5156.

[2] Bezerianos.A, Laskaris.N,et al,(1996), "Selective noise filtering of high resolution ECG through Wavelet Transform", IEEE Pub, pp 637-640.

[3] Brigham.E.O,(1998),“The Fast Fourier Transform and its Applications”,Prentice-Hall.

[4] Burrus.C.S, Gopinath.R.A,et al, (1997), ,'Introduction to Wavelets and Wavelet Transforms", a Primer, Prentice Hall Inc.

[5] Chavan M. S., Aggarwala.R.A,(2008), "Interference reduction in ECG using digital FIR filters based on Rectangular window", WSEAS Transactions on Signal Processing, Issue 5, Volume 4, pp 340-49.

[6] Datar A., Jain A.,Sharma, P.C,(2009),"’Performance of Blackman window famiy in M-channel cosine modulated filter bank for ECG signal", Multimedia, Signal Processing and Communication Technologies. IMPACT '09. International, IEEE Conference,Aligarh,ISBN: 978-1-4244-3602-6, pp $98-101$.

[7] DagStanneby,(2001), “Digital Signal Processing-DSP Application”, Butterworth Heinemann, Oxford.

[8] Ercelebi.E,(2004), "Electrocardiogram signals de-noising using lifting-based discrete wavelet transform", Comput. Bio. Med. 34 (6) 479-493 .

[9] Ferdjallah.M, Barr.R.E,(1990), "Frequency Domain Digital filtering techniques for removal of Powerline noise with application to the Electrocardiogram", pp 475-89.

[10] Herrera-Bendezd L.G, Garcia.J,et al,(1992), "Real time digital filters for ECG signals:Evaluation and new designs", IEEE pub., pp-133-136.

[11] Jitendra Kumar Verma,(2015), "Enhancement of ECG signal by DFT using Fast Fourier Transform(FFT) algorithm", International Journal of Current Engineering and Technology, Vol 5, No 3, E-ISSN 2277-4106, P-ISSN 2347-5161. 
[12] Manoj, Vinod Kumar,et al,(2014), "Comparative study of Different filters with several Window Techniques using Wavelet for removing Noise from ECG signal", International Journal of Research in Advent Technology,Vol 2, No 5, E-ISSN 2321-9637.

[13] MayurKumarvChhipa,(2013), "Performance analysis of various transforms based methods of ECG data", International Journal of Scientific and Research Publications, Vol. No. 03, Issue 5, ISSN 2250-3153.

[14] Mbachu . C. B,(2011), "Processing ECG signal with Kaiser Window - Based FIR digital filters", International Journal of Engineering Science and Technology, Vol 3, No 8, ISSN 09755462.

[15] Pranob K. Charles and Rajendra Prasad K,(2011), “A Contemporary Approach For ECG Signal Compression Using Wavelet Transforms", Signal and Image Processing: An International Journal (SIPIJ), Vol. 2, No. 1, 178-183.

[16] Preeti Patel,(2014), "Efficient filtering techniques of ECG signal using FIR low pass filter with various Window Techniques", International Journal of Advanced Research in Computer Science and Software Engineering, Vol 4, Issue 7, ISSN 2277-128X.

[17] Rovin Tiwari, (2014), "ECG analysis based on Window filtering approach using Empirical Mode Decomposition Techniques(EMD)", IOSR Journal of Electronics and Communication Engineering, Vol 9, Issue 3,e-ISSN 2278-2834, p-ISSN 2278-8735.

[18] Samarjeet Singh and Uma Sharma, “MATLAB based Digital IIR filter design”, International Journal Electronics and Computer Science Engineering, Vol. No. 01, ISSN 2277-1956,pp.7483 